\title{
Co-existence of Alport syndrome and C3 glomerulonephritis in a proband with family history
}

Yin Ding, Xuanli Tang, Yuanyuan Du, Hongyu Chen, Dongrong Yu, Bin Zhu and Bohan Yuan*

\begin{abstract}
Background: Alport syndrome and C3 glomerulonephritis (C3GN) are rare kidney diseases, frequently responsible for familial haematuria, proteinuria, and renal impairment. With the rapid development of molecular genetic testing, Alport syndrome causes have been restricted mostly to variants in the COL4A5 or COL4A3/COL4A4 genes. Moreover, a broad range of genetic contributors in the complement and complement-regulating proteins are definitely implicated in the pathogenesis of C3GN.
\end{abstract}

Methods: We sought a family with persistent microscopic haematuria associated with renal failure. Clinicopathologic and follow-up data were obtained, and molecular genetic testing was used to screen for pathogenic variants.

Results: We describe a three-generation family with Alport syndrome showing a dominant maternal inheritance. Notably, renal biopsy showed the concurrent histological evidence of C3GN in the proband harbouring an uncommon heterozygous variation in CFHR5, c.508G > A. The alteration leads to replacement of a highly conserved residue at position 170 of the $\beta$-strand subunit of CFHR5 (p.Val170Met). In silico analysis showed that the variation was predicted to deregulate complement activation by altering the structural properties and enhancing C3b binding capacity to compete with Complement Factor H (CFH), which was in line with experimental data previously published.

Conclusions: The comorbidity findings between Alport syndrome and C3GN indicate an underlying overlap and require further study.

Keywords: Rare renal disease, Alport syndrome, C3GN, CFHR5 p.Val170Met

\section{Background}

In China, a rare disease is defined as a condition that affects fewer than 500,000 people [1]. Approximately $80 \%$ of rare diseases have an identified genetic background with substantial geographic or ethnic variations in incidence [2].

Genetic analysis was first used in nephrology for the identification of a causal mutation for Alport syndrome

*Correspondence: yuanbohan26@163.com

Department of Nephrology (Key Laboratory of Management

of Kidney Disease in Zhejiang Province), Hangzhou TCM Hospital

Affiliated to Zhejiang Chinese Medical University, Tiyuchang Road 453,

Hangzhou 310007, People's Republic of China in 1990 [3]. Alport syndrome is a rare hereditary disorder in which the glomerular basement membrane (GBM) has an abnormal collagen IV composition. It is characterized by haematuria, progressive renal failure, sensorineural deafness, anterior lenticonus and retinal flecks [4]. To date, the causes of familial haematuria nephropathies have been restricted mostly to variants in the COL4A5 or COL4A3/COL4A4 genes, responsible for X-linked or autosomal inheritance [5-7]. Moreover, analysis of data suggests that familial microscopic haematuria attributable to the complement factor $\mathrm{H}$-related protein 5 (CFHR5) gene is also associated with a rare renal disease termed C3 glomerulonephritis (C3GN) [8-12], defined as glomerular $\mathrm{C} 3$ position in the absence of significant 
amounts of immunoglobulin, $\mathrm{C} 1 \mathrm{q}$, and $\mathrm{C} 4$, and without intramembranous glomerular basement membrane dense deposits.

Herein, we report a family with dominant maternal transmitted inheritance, in which five out of eight members (I:1, II:1, II:4, III:1, and III:2) exhibited microscopic haematuria and proteinuria and two out of the 5 patients (II:1 and III:1) developed end-stage renal disease (ESRD). The diagnosis of Alport syndrome was confirmed in the proband (III:1), his mother (II:1), and his maternal aunt's daughter (III:2) based on all available data. Furthermore, renal biopsy showed the comorbidity of Alport syndrome and C3GN in the proband carrying an uncommon heterozygous c.508G > A (p.Val170Met) variant in the CFHR5 gene. Furthermore, the functional effect of the p.Val170Met variation was exhaustively evaluated.

\section{Methods}

\section{Family pedigree and clinical investigations}

In this study, a family with persistent microscopic haematuria associated with renal failure was recruited from Hangzhou, Zhejiang Province, China. All affected members were regularly followed up at the out-clinic until May 12, 2020. A brief history and physical examination were performed and blood, urine, skin, kidney samples were collected for determination of serum creatinine concentration, estimated glomerular filtration rate (eGFR), urinalysis, $\mathrm{C} 3 / \mathrm{C} 4$ levels and histological manifestation.

\section{Renal and skin biopsies processing}

Biopsy specimens for light microscopy were fixed in alcohol formaldehyde acetic solution, embedded in paraffin, and cut into $2-\mu \mathrm{m}$-thick sections. Sections were stained with Masson trichrome, hematoxylin and eosin, periodic acid-Schiff, and silver methenamine. Samples for immunofluorescence study were stained with fluorescein isothiocyanate-conjugated polyclonal antibodies to human IgA, IgG, IgM; C3; light chains ( $\kappa$ and $\lambda$ ); $44, \mathrm{C} 1 \mathrm{q}$; and $\alpha 5$ chains of type IV collagen. Antigen was retrieved by EDTA solution as well as gastric enzyme, and Elivision method was applied in IHC detection. Specimens for electron microscopy were fixed in glutaraldehyde and embedded in epon. All reports were reviewed by two pathologists.

\section{Targeted exon sequencing and Sanger sequencing}

Genomic DNA was isolated from peripheral blood of a three-generation pedigree of eight members using the Wizard Genomic DNA Purification Kit (Promega, USA) following the manufacturer's instructions. Coding exons reference sequences of the individuals (II:1, II:2, III:1 and III:2) were targeted for the Illumina high-throughput sequencing, and reads were aligned to the human genome reference sequence (hg19/GRCh37). The approximately $95 \%$ of reads were mapped to the target regions at an average of $20 \times$. We examined copy number, rare, and common variants. All the disease-related sites were selected and Sanger sequencing was further performed in all eight members. Primers were indigenously designed using the primer premier 5.0 program (Lalitha, 2000) and shown in Additional file 1: Table S1. The purified PCR products were directly sequenced using an ABI BigDye Terminator v3.1 Cycle Sequencing Kit. The analyses were completed on an ABI-3500Dx Genetic Analyzer (Applied Biosystems).

\section{In silico evaluation of pathogenicity}

The ProtParam tool was used to compute various physical and chemical parameters including the molecular weight and theoretical pI. The complete amino acid sequence data of the human protein CFHR5 (GenBank Accession: AAI11774.1) were obtained from the NCBI (National Center for Biotechnology Information) and alignments done by the EMBL-EBI (European Bioinformatics Institute). The SIFT (Sorting Intolerant From Tolerant), SNAP (Screening for Non-acceptable Polymorphisms), PolyPhen-2 (Polymorphism Phenotyping v2), and Mutation Taster were utilized to evaluate possible biologic effects of genetic aberration impact on protein structure.

\section{Variant interpretation}

In 2015, standards and guidelines for the interpretation of sequence variants were updated by the American College of Medical Genetics and Genomics (ACMG), the Association for Molecular Pathology (AMP) and the College of American Pathologists (CAP) [13]. This report recommends the use of specific standard terminology: 'pathogenic', 'likely pathogenic', 'uncertain significance,' 'likely benign', and 'benign' to describe variants.

In brief, an allele frequency in a control population that is greater than expected for disorder or with lack of segregation among affected individuals was considered strong support for a benign interpretation or, if over $5 \%$, it is considered as 'benign'. Novel or rare variants that lead to splicing defect or amino acid change coupled with multiple lines of computational evidence, or cosegregate with disease, were classified as 'pathogenic' or 'likely pathogenic', and variants with well-established in vitro or in vivo functional studies that indicate a damaging effect were labelled 'pathogenic'. Variants of 'uncertain significance' are more common: other criteria shown above are not met or the criteria for benign and pathogenic are contradictory. 


\section{Homology modelling of human CFHR5/C3b complex}

Docking procedure has been described in more detail previously [14]. Starting from residues $23-569$, the 3D homology models of CFHR5 were first generated by iterative threading assembly refinement (I-TASSER) server [15], where the one with the highest C-score was selected to be further refined by Fragment-Guided MD simulation (FG-MD) [16]. Potential energy of refined protein was calculated by "Calculate Energy" protocol of Discovery Studio (DS) 3.0. The crystal structure of ligand C3b (PDB ID: 2WII) was taken from protein data bank (PDB).

By applying "Prepare Protein" protocol of DS, energy minimization was performed to clean the protein molecule by adding missing atoms, inserting missing loops, assigning charges and fixing CHARMm force fields. A total of 2000 docking poses were generated by ZDOCK
(CHARMm-based DOCKER) protocol and incorporated. The top1 model was collected as the probable complex structure. Hydrogen-bonding networks can be displayed by Molecular Operating Environment (MOE). Binding free energy of protein-protein interaction was estimated by the Calculate Mutation Energy (Binding) protocol within DS.

\section{Results}

\section{Clinical presentation and follow-up}

We investigated a three-generation Han Chinese family characterized by persistent microscopic haematuria associated with renal failure (Fig. 1a). The proband (III:1), a 11-year-old boy, was admitted to the hospital for haematuria and proteinuria. On admission, 24-h urine protein was $6.36 \mathrm{~g} /$ day and serum albumin was $24.4 \mathrm{~g} / \mathrm{L}$.

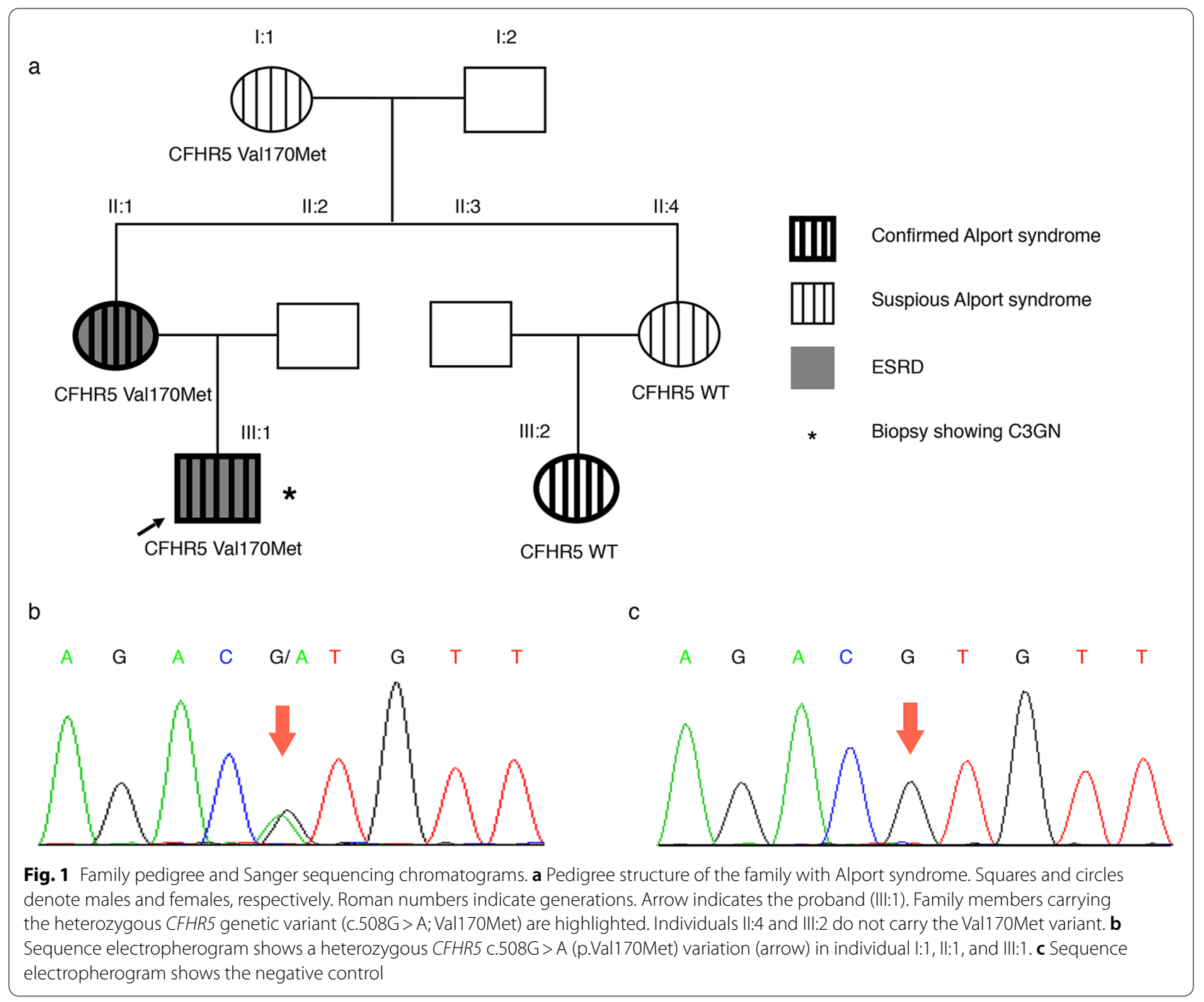


Microscopic urinary analysis showed urine sediment containing ++ to +++ red cells per high-power field. His blood urea nitrogen level was $15.73 \mathrm{mmol} / \mathrm{L}$, serum creatinine level was $266 \mu \mathrm{mol} / \mathrm{L}$ and eGFR by CKD-EPI equation was $30.3 \mathrm{~mL} / \mathrm{min}$. Bilateral renal ultrasound revealed abnormal changes with strong and unevenly distributed echoes, and unclear demarcation between the renal cortex and parenchyma. Sensorineural hearing impairment and vision abnormalities, as well as other clinical signs, were not detected.

During follow-up, the proband was started on haemodialysis 3 months later and received a kidney transplant after one year. The proband (III:1) had a family history of haematuria, as his mother (II:1) presented with haematuria, proteinuria, and developed ESRD before the age of 33 years. His maternal grandmother (I:1), maternal aunt (II:4), and aunt's daughter (III:2) also had urinary abnormalities characterized by asymptomatic haematuria and proteinuria with normal eGFR. His father (II:2), his maternal grandfather, and aunt's husband were phenotypically normal.

To confirm the clinical diagnosis, renal biopsy of the proband was performed. The biopsy sample contained 15 glomeruli on light microscopy: global glomerular sclerosis in four glomeruli, segmental sclerosis in three other glomeruli (Fig. 2a), and four crescents were found (Fig. 2b). Diffuse and global mild-moderate proliferation of mesangial cells and mesangial matrix were shown in the remaining glomeruli (Fig. 2c). Besides, interstitial fibrosis/tubular atrophy containing lipid-laden foam
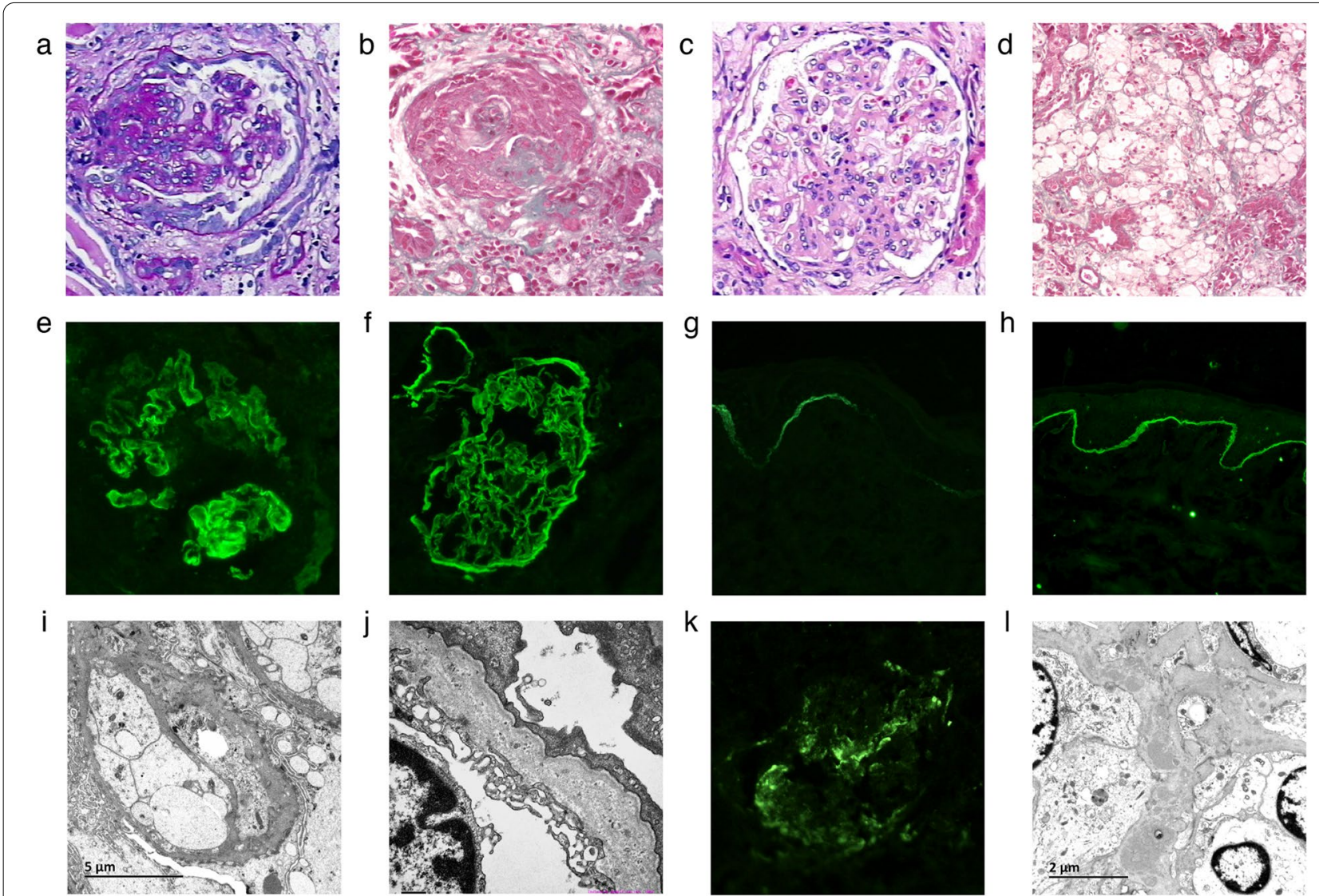

Fig. 2 The pathological features of kidney and skin samples in the proband (III:1), his mother (II:1), and His affected aunt's daughter (III:2). Light micrograph: the proband shows segmental sclerosis (a, PAS $\times 200)$, crescent formation (b, MA $\times 200)$, diffuse and global mild-moderate mesangial proliferation (c, HE × 200), and interstitial fibrosis/tubular atrophy containing lipid-laden foam cells (d, MA × 200). Immunofluorescent stain: the proband reveals partial loss for the collagen IV a5 chain in GBM and complete absence in Bowman's capsule and distal tubule basement membrane (e, IF $\times 400)$, compared with control (f, IF $\times 400)$. A skin biopsy of his mother demonstrates segmental absence of the collagen IV a5 chain within the epidermal basement membrane (g, IF $\times 200)$, compared with control (h, IF $\times 200)$. Electron micrograph: segmental uneven thickness with unsmooth spiculation of the dense layer in the GBM is found in the proband (i, $\times 6000)$, and uneven thickness with a laminated appearance is shown in his aunt's daughter $(\mathbf{j}, \times 15,000)$. Remarkably, a marked C3 staining is shown in the proband to locate along the mesangium by immunofluorescence analysis ( $\mathbf{k}$, IF $\times 200)$ with the corresponding electron-dense deposits under the electron microscopy $(\mathbf{I}, \times 10,000)$ 
cells were obvious (Fig. 2d). Immunofluorescence studies showed patchy GBM expression of a5 (IV) collagen and complete absence in Bowman's capsule and distal tubular basement membrane (BM) (Fig. 2e), compared with control (Fig. 2f). A skin biopsy was performed on his mother (II:1), which revealed that there was segmental absence of the collagen IV a5 chain within the epidermal basement membrane (Fig. 2g), compared with control (Fig. 2h).

Electron microscopy identified pathological characteristics accompanied with noticeably segmental uneven thickness with unsmooth spiculation and suspected lamination of the dense layer within the basement membrane in the proband (Fig. 2i). His affected aunt's daughter (III:2) had a renal biopsy at age 13, showing segmental uneven thickness with a laminated appearance of the dense layer in GBM (Fig. 2j).

Notably, a marked C3 deposition $(++$ to +++$)$ in the mesangial region was detected in the proband under immunofluorescence microscopy (Fig. 2k), with the corresponding lump electron-dense deposits and mildmoderate mesangial proliferation observed with electron microscopy (Fig. 2l). Immunofluorescence examination revealed none or trace positivity of $\operatorname{IgA}, \operatorname{IgG}, \operatorname{IgM}, \mathrm{C} 4$, C1q, Fib, $\kappa$ and $\lambda$ fragments.

\section{Findings on targeted exome-based next-generation sequencing (NGS) and Sanger sequencing}

To make a precise diagnosis for the true pathogenic mechanism affecting the family, we performed whole exome-based NGS and further confirmed the results with Sanger sequencing. Genetic analysis identified the same heterozygous c.508G $>$ A coding variant (p.Val170Met) in exon 6 of the CFHR5 gene in three affected individuals: the proband (III:1), his mother (II:1) and his maternal grandma (I:1) (Fig. 1b). The variant presents low frequency in the general population (1000 Genomes: minor allele frequency $[\mathrm{MAF}]=0.1 \%$ ), and the mutated A allele carriers are all heterozygous. Compared across ethnicities, this variant is more frequent in East Asians (1000 Genomes: $0.6 \%$ ), especially in the Chinese Han population (1000 Genomes: $1 \%$ ). The nonsynonymous alteration leads to the replacement of a valine, strictly conserved among organisms (Fig. 3a), by a methionine residue. The screening with four publicly available programs (SIFT, SNAP, PolyPhen-2, and Mutation Taster) independently predicted that the replaced amino acid was "damaging" to the protein structure/function (SIFT score 0.004; SNAP score 20; PolyPhen2 score 0.999; Mutation Taster: might be affected).

No suspicious disease-causing variants were detected in the genes COL4A3, COL4A4, or COL4A5 that encode the $\alpha 3, \alpha 4$, or $\alpha 5$ chains of type IV collagen, respectively.

\section{In silico functional prediction for CFHR5 C.508G > A,} p.Val170Met

CFHR5 is a single-chain polypeptide composed of nine complement control protein domains (also known as CCPs). The residue p.Val170Met in CFHR5 occurs in the $\beta$-strand region of the CCP3. The isoelectric point (pI) is found to be the same for both wild type and mutant proteins (pI 6.8). Molecular weight of the mutant (64.45 kDa) protein is similar to that of wild-type protein $(64.42 \mathrm{kDa})$. In the native structure, the Val residue, located in the buried surface, is not involved in any intramolecular interactions. As substituted by Met170, the original intramolecular hydrogen-bonding distance between the side chain of its neighbouring residues (Leu171 and Val187) changes (Fig. 3b). DS 3.0 analysis predicted the potential energy of the mutant-type protein is $-15180.97 \mathrm{kcal} / \mathrm{mol}$ compared to $-15133.86 \mathrm{kcal} / \mathrm{mol}$ for the wild-type one, which implied that p.Val170Met could lead to an increase in conformational stability of the CFHR5 protein.

As with Complement Factor $\mathrm{H}(\mathrm{CFH})$ and other Complement Factor $\mathrm{H}$ Related Proteins (CFHRs), CFHR5 regulates the complement cascade by binding and interacting with the macromolecular protein ligand C3b. The $\mathrm{C} 3 \mathrm{~b}$ fragment is a glycoprotein composed of the modified C3- $\alpha$ chain $\left(C 3 \alpha^{\prime}\right)$ and the intact $C 3-\beta$ chain $(C 3 \beta)$. We then simulated the probable native $C F H R 5 / \mathrm{C} 3 \beta$ complex structure. As it can be seen in Fig. 3c, the three-dimensional (3D) model demonstrated that Val170 is not the binding site for protein C3b. However, further binding free-energy calculation showed that p.V170M slightly increases the binding affinity of $C F H R 5 / C 3 \beta$ complex by $-0.76 \mathrm{kcal} / \mathrm{mol}$.

\section{Discussion}

Alport syndrome is characterized by haematuria, renal failure, and extra-renal alterations, such as: hearing loss, lenticonus, and retinal flecks [4-7]. The disease is caused by changes in the collagen type IV chains, resulting in damage to the basement membrane of several organs. Approximately $85 \%$ of families have X-linked inheritance with mutations in COL4A5 gene [3, 17-19], and most of the other affected families have autosomal recessive disease with alterations in both copies of COL4A3 or COL4A4 [20-22]. Autosomal dominant inheritance is very rare and results from heterozygous COL4A3 or COL4A4 variants.

In our study, we comprehensively examined the history of a three-generation family. The proband (III:1) presented progressive deterioration of renal function with persistent haematuria and proteinuria, and underwent a kidney transplant at age 12 . His family history was significant, as her mother progressed to ESRD, over a 
a

\begin{tabular}{ll|l} 
Species & aa sequence & 170 \\
Homo sapiens & VDAQPKKESYKVGD & LKFSCRKNLIRVGSDSVQC \\
Acinonyx jubatus & IHVYPKQEKYTPGD & LQCSCRQGLVRVGPDSVQC \\
Ursus arctos horribilis & VDVYPRQVKYKAGD & LQF SCGQRLTRVGPDSVQC \\
Piliocolobus tephrosceles & VDAKPEKESYEVGD & LTFSCRKNLVRVGPDSVQC \\
Callorhinus ursinus & LDVYPRKVKYNAGD & LQFSCGQRLKRVGPDSVQC \\
Canis lupus dingo & VGVYPRRVRYKTGE & LQFSCEQGLTGVGPASVQC \\
Theropithecus gelada & VDAKPEKESYKVGD & LKFSCRKNLKRVGPDSVQC \\
Pan paniscus & VDAQPKKESYKVGD & LKFSCRKNLIRVGSDSVQC \\
Macaca nemestrina & VDAKPKKESYKVGD & LKFSCRKNLTRVGPESVQC \\
Felis catus & IHVYPKQEKYTPGD & LQFSCRQGLVRVGPDSVQC \\
Canis lupus familiaris & VGVYPRRVRYKTGE & LQFSCEQGLTGVGPASVQC
\end{tabular}

C

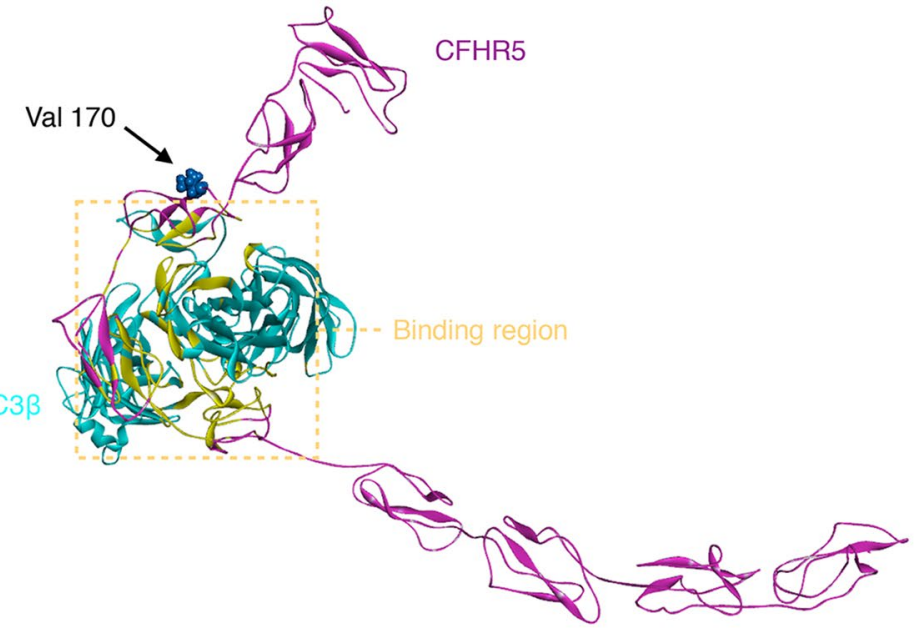

b

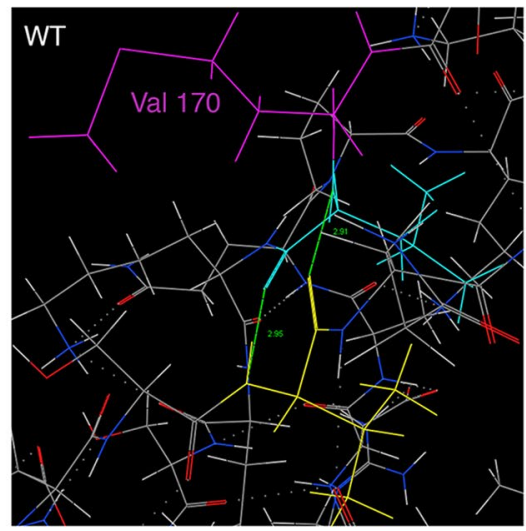

...... H-bond

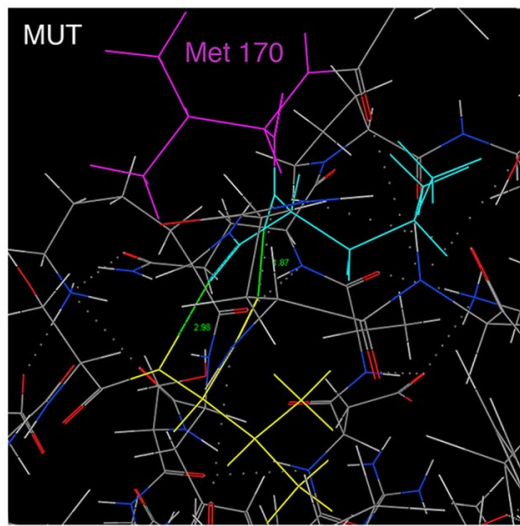

Fig. 3 Functional characterization of the CFHR5 p.Val170Met variant. a Alignment of the CFHR5 protein in different species shows the conservation of the $\mathrm{V} 170$ residue. The concerned amino acids are boxed. $\mathbf{b}$ Generated models show the discrimination of local intramolecular hydrogen-bonding interactions between Val170 and Met 170 by MOE. Hydrogen bonds are shown with a fluorescent green dotted line representation. $\mathbf{c}$ Structure of CFHR5 in complex with the ligand C3b- $\beta$ chain (C3 $\beta$ ) were generated with the DS 3.0. CFHR5 is denoted by pink, C3 $\beta$ (residues 1-642, PDB 2WII) is shown in turquoise. The binding regions of the CFHR5/C3 3 complex are presented in yellow. The val residue 170 of CFHR5 is highlighted with a CPK model

10-year follow-up period of haematuria and proteinuria. Besides, his maternal grandma (I:1), maternal aunt (II:4), and aunt's daughter (III:2) had asymptomatic microscopic haematuria $(<30 / \mathrm{HP})$ and proteinuria $(<0.5 \mathrm{~g} / \mathrm{d})$ with normal renal function. Characteristic pathological changes were observed in renal biopsy specimens from the proband (III:1) and his maternal aunt's daughter (III:2), including segmental uneven thickness with lamellation and splitting of the dense layer in GBM under electron microscopy. In addition, immunostaining showed nearly complete loss for a5 (IV) collagen chain in GBM and complete absence in Bowman's capsule and distal tubule basement membrane in the proband. His mother (II:1) exhibited segmental distribution of a5 (IV) expression in the skin BM. According to the diagnostic algorithm published in 2013 [7], the diagnosis of Alport syndrome was established in the proband (III:1), and his mother (II:1) and his maternal aunt's daughter (III:2) based on pedigree study, clinical manifestation, and skin/ renal biopsy.

The COLAA3 and COL4A4 genes are located on chromosome 2, while the COL4A5 gene is located on the $\mathrm{X}$ chromosome, these genes encode the collagen IV a3, a4, and a5 chains, respectively. In normal individuals, the collagen IV a3a4a5 chains are highly expressed and co-distributed within the mature kidney (GBM and distal tubular BM), cochlea, and eye, and the collagen IV a5a5a6 network occurs in the skin BM and kidney (Bowman's capsule) [5]. X-linked Alport syndrome accounts for the majority of Alport syndrome cases, arising from mutations in the COL4A5 gene. In the proband and his mother, morphologic phenotype demonstrated reduced and depleted expression of the collagen IV a5 chain in kidney and/or skin. Further clinical and genealogical 
study indicated that the affected proband (III:1) had much more severe disease than the females [his mother (II:1), maternal grandma (I:1), maternal aunt (II:4) and aunt's daughter (III:2)] in the family, and male-to-male transmission was absent. Hence, the mode of inheritance was suspected to be X-linked.

Molecular genetic testing is one of the criteria for the diagnosis of Alport syndrome with a high sensitivity and specificity $(>90 \%)[7,23,24]$. High-throughput nextgeneration sequencing (NGS) technology can improve the diagnosis of Alport syndrome by providing molecular confirmation of COL4A3, COL4A4, or COL4A5 mutations. We performed whole-exome sequencing for four individuals (II:1, II:2, III:1, and III:2); however, we did not identify any variant that could be possibly diseasecausing in the three type IV collagen genes. It is possible that a small proportion of variants may have been missed owing to unfavourable insurance coverage or for other reasons. Alternatively, there could be a rare deep intronic variant affecting splicing and only detectable by RNA analysis. In addition, pathogenic genes in families with Alport syndrome may not be confined to a few regions. Known COL4A3, COL4A4, and COL4A5 genes are scattered throughout many exons, making it difficult to develop predictive genetic tests.

Notably, a marked C3 staining with the corresponding electron-dense deposits along the mesangium was detected by immunofluorescence and electron microscope analysis of the kidney biopsy in the proband (III:1). Serum C3 was reduced in his mother (II:1), but it was normal in the proband and other affected family members. Excessive glomerular C3 fragment deposition without immunoglobulin, mesangial proliferation and crescent formation showed some morphological features of C3GN. In addition, the proband and his mother were affected more severely than other members. In the pedigree, familial C3GN was suspicious. The findings above prompted us to further investigate the complement system in the index family. A broad range of genetic contributors are definitely implicated in the pathogenesis of C3GN [25-27], so we screened a set of complement genes.

We identified a heterozygous missense variant c.508G > A (p.V170M; rs201073457) in the complement regulatory gene CFHR5 in three members of this family (I:1, II:1 and III:1). CFHR5 colocalizes with complement-containing glomerular immune deposits in a variety of glomerular pathologic states [28]. Based on genetic studies, copy number variations in CFHR5 are implicated in $\mathrm{C} 3 \mathrm{GN}[8,29,30]$, but significant enrichment of disease-associated rare variants is less [31]. The c.508G > A in the gene CFHR5 generates a nonsynonymous alteration at amino acid position 170 (Val in wild-type and Met in mutant), which is strictly conserved among organisms. In silico programs (SIFT, SNAP, PolyPhen-2, and Mutation Taster) independently indicated the CFHR5 c.508G > A to be potential functional variant. The exact mechanism of complement regulation is unknown, but is probably related to the C3b binding capacity. Then, we simulated 3D homology-modelled structures, which indicated mutant-type CFHR5 increased conformational stability and induced an increase in the C3b-binding affinity. Meanwhile, this alteration has been previously reported in a patient with aHUS [32] and verified to exhibit significantly higher $\mathrm{C} 3 \mathrm{~b}$ binding capacity compared with wild-type CFHR5 in in vitro functional assays [33]. Further replication studies are important and additional functional studies are required to reveal the exact biologic impacts in C3GN.

The above computational and functional assessments indicated that CFHR5 c.508G > A may have contributed to the C3GN phenotype in the proband. Then, we applied the ACMG standards for variant classification [13], based on typical types of evidence (e.g., population data, computational and predictive data, functional data, segregation data and de novo data). The CFHR5 c.508G > A variant is clearly PP3 [multiple lines of computational evidence support a deleterious effect on the gene or gene product (conservation, evolutionary, splicing impact, etc.)], likely PS3 (well-established in vitro or in vivo functional studies supportive of a damaging effect on the gene or gene product). Unfortunately, the evidence in favour of this variant being 'pathogenic' or 'likely pathogenic' is not substantial.

Meanwhile, it is not to be ignored that, C3GN is an ultra-rare kidney disease with an incidence of approximately one case per million per year [34]. Compared across ethnicities, the CFHR5 c.508G >A variant is relatively frequent in East Asians (1000 Genomes: $0.6 \%)$, especially in the Chinese Han population (1000 Genomes: $1 \%)$. Though genetic investigation revealed the uncommon CFHR5 missense variant in three affected individuals: the proband, his mother and his maternal grandma, the last two did not undergo renal biopsy. Currently, it is clearly BS1 (allele frequency is greater than expected for disorder), and clearly BS4 (lack of segregation in affected members of a family), which comes out that the CFHR5 c.508G > A variant is classified as 'benign'.

\section{Conclusions}

Our study reports a pedigree characterized by microscopic haematuria and variety in the speed of onset of renal failure. The diagnosis of Alport syndrome is 
confirmed in the proband (III:1), his mother (II:1) and his maternal aunt's daughter (III:2). Most notably, the proband revealed morphological features of C3GN based on genetic complement dysregulation background with concurrent histological evidence of Alport syndrome. Cases of Alport syndrome complicated with C3GN has never been reported, and underlying overlap requires further study.

\begin{abstract}
Abbreviations
CFHR5: Complement factor H-related protein 5; C3GN: C3 glomerulonephritis; ESRD: End-stage renal disease; eGFR: Estimated glomerular filtration rate: MAF: Minor allele frequency; BUN: Blood urea nitrogen; sCr: Serum creatinine; GBM: Glomerular basement membrane; BM: Basement membrane; NGS: Next-generation sequencing; CKD: Chronic kidney disease; WT: Wild type; MT: Mutant type.
\end{abstract}

\section{Supplementary Information}

The online version contains supplementary material available at https://doi. org/10.1186/s40001-021-00543-5.

Additional file 1: Table S1. Primers of Sanger sequencing for CFHR5 C.508G > A.

\section{Acknowledgements}

We thank all the individuals who participated in this study.

\section{Authors' contributions}

Experiment performance, data analysis and interpretation, figure preparation and manuscript writing:YD. Histological and laboratory analysis and interpretation: XT, YD. Study design, clinical analysis and interpretation: HC, DY, BZ. Patient recruitment, counseling and follow-up, study design: BY. All authors read and approved the final manuscript.

\section{Funding}

This work was supported by grants from National Natural Science Foundation of China (No. 81973760), Project of Science and Technology Agency of Zhejiang Province of China (No. LGF19H270007) and Project of Administration of Traditional Chinese Medicine of Zhejiang Province of China (No. 2019ZA086) which paid for language polishing service. The funding body had no role in the design of the study, collection, analysis and interpretation of data and in writing the manuscript.

\section{Availability of data and materials}

The datasets used and/or analysed during the current study are contained within the manuscript and the appendix, and available from the corresponding author on reasonable request. The totality of the data cannot be shared based on patient confidentiality concerns.

\section{Declarations}

\section{Ethical approval and consent to participate}

All of the procedures performed in studies involving human participants were carried out in accordance with the ethical standards of the Ethics Committee of the Hangzhou hospital of traditional Chinese medicine (institutional review board Approval Number: 2020KY055) and with the 1964 Declaration of Helsinki and its later amendments or comparable ethical standards. Written informed consent forms for participation in the study were obtained, where participants are children (under 16 years old) from their parent.

\section{Consent for publication}

Not applicable.

\section{Competing interests}

The authors declare that they have no competing interests.

Received: 30 May 2021 Accepted: 28 June 2021

Published online: 08 July 2021

\section{References}

1. Liu BC, He L, He G, et al. A cross-national comparative study of orphan drug policies in the United States, the European Union, and Japan: towards a made-in-China orphan drug policy. J Public Health Pol. 2010;31:407-21.

2. Devuyst $\mathrm{O}$, Knoers NVAM, Remuzzi G, et al. Rare inherited kidney diseases: challenges, opportunities, and perspectives. Lancet. 2014;383:1844-59.

3. Barker DF, Hostikka SL, Zhou J, Chow LT, et al. Identification of mutations in the COLAA5 collagen gene in alport syndrome. Science. 1990:248:1224-7.

4. Kruegel J, Rubel D, Gross O. Alport syndrome-insights from basic and clinical research. Nat Rev Nephrol. 2013;9:170-8.

5. Hudson BG, Tryggvason K, Sundaramoorthy M, Neilson EG, et al. Alport's syndrome, Goodpasture's syndrome, and type IV collagen. N Engl J Med. 2003;348:2543-56.

6. Kashtan CE, Ding J, Garosi G, et al. Alport syndrome: a unified classification of genetic disorders of collagen IV alpha345: a position paper of the Alport syndrome classification working group. Kidney Int. 2018;93:1045-51.

7. Savige J, Gregory M, Gross O, et al. Expert guidelines for the management of alport syndrome and thin basement membrane nephropathy. J Am Soc Nephrol. 2013;24:364-75.

8. Athanasiou Y, Voskarides K, Gale DP, et al. Familial C3 glomerulopathy associated with CFHR5 mutations: clinical characteristics of 91 patients in 16 pedigrees. Clin J Am Soc Nephro. 2011;6:1436-46.

9. Besbas N, Gulhan B, Gucer S, et al. A novel CFHR5 mutation associated with C3 glomerulonephritis in a Turkish girl. J Nephrol. 2014:27:457-60.

10. Gale DP, de Jorge EG, Cook HT, Martinez-Barricarte R, et al. Identification of a mutation in complement factor $\mathrm{H}$-related protein 5 in patients of Cypriot origin with glomerulonephritis. Lancet. 2010;376:794-801.

11. Terence Cook H. Evolving complexity of complement-related diseases: C3 glomerulopathy and atypical haemolytic uremic syndrome. Curr Opin Nephrol Hypertens. 2018;27:165-70.

12. Pickering $M C$, et al. C3 glomerulopathy: consensus report. Kidney Int. 2013;84:1079-89.

13. Richards S, Aziz N, Bale S, et al. Standards and guidelines for the interpretation of sequence variants: a joint consensus recommendation of the American College of Medical Genetics and genomics and the association for molecular pathology. Genet Med. 2015;17:405-24.

14. Ding Y, Zhao W, Zhang T, et al. A haplotype in CFH family genes confers high risk of rare glomerular nephropathies. Sci Rep. 2017;20(7):6004 https://doi.org/10.1038/s41598-017-05173-8.

15. Yang J, Yan R, Roy A, et al. The I-TASSER Suite: protein structure and function prediction. Nat Method. 2015;12:7-8.

16. Zhang J, Liang Y, Zhang Y. Atomic-level protein structure refinement using fragment-guided molecular dynamics conformation sampling. Structure. 2011;19:1784-95.

17. Fernandez-Rosado F, Campos A, Alvarez-Cubero MJ, et al. Improved genetic counseling in alport syndrome by new variants of COL4A5 gene. Nephrology. 2015;20:502-5.

18. Jais JP, Knebelmann B, Giatras I, et al. X-linked alport syndrome: natural history in 195 families and genotype-phenotype correlations in males. J Am Soc Nephrol. 2000;11:649-57.

19. Yoshioka K, Hino S, Takemura T, et al. Type IV collagen alpha 5 chain. Normal distribution and abnormalities in X-linked alport syndrome revealed by monoclonal antibody. Am J Pathol. 1994;144:986-96.

20. Mencarelli MA, Heidet L, Storey H, et al. Evidence of digenic inheritance in alport syndrome. J Med Genet. 2015;52:163-74.

21. Mochizuki T, Lemmink HH, Mariyama M, et al. Identification of mutations in the alpha 3(IV) and alpha 4(IV) collagen genes in autosomal recessive alport syndrome. Nat Genet. 1994;8:77-81. 
22. Van der Loop FT, Heidet L, Timmer ED, van den Bosch BJ, et al. Autosomal dominant Alport syndrome caused by a COL4A3 splice site mutation. Kindey Int. 2000;58:1870-5.

23. Deltas C, Pierides A, Voskarides K. The role of molecular genetics in diagnosing familial haematuria(s). Pediatr nephrol. 2012;27:1221-31.

24. Gale DP. How benign is haematuria? Using genetics to predict prognosis. Pediatr Nephrol. 2013:28:1183-93.

25. latropoulos P, Noris M, Mele C, et al. Complement gene variants determine the risk of immunoglobulin-associated MPGN and C3 glomerulopathy and predict long-term renal outcome. Mol Immunol. 2016;71:131-42.

26. Servais A, Noel LH, Roumenina LT, et al. Acquired and genetic complement abnormalities play a critical role in dense deposit disease and other C3 glomerulopathies. Kidney Int. 2012;82:454-64.

27. Sethi S, Fervenza FC. Membranoproliferative glomerulonephritis-a new look at an old entity. N Eng J Med. 2012;366:1119-31.

28. Murphy B, Georgiou T, Machet D, et al. Factor H-related protein-5: a novel component of human glomerular immune deposits. Am J Kidney Dis. 2002;39:24-7.

29. Gale DP, Maxwell PH. C3 glomerulonephritis and CFHR5 nephropathy. Nephrol Dial Transpl. 2013:28:282-8.

30. Zipfel PF, Wiech T, Stea ED, Skerka C. CFHR gene variations provide insights in the pathogenesis of the kidney diseases atypical hemolytic uremic syndrome and c3 glomerulopathy. J Am Soc Nephrol. 2020;31:241-56.

31. Levine AP, Chan MMY, Sadeghi-Alavijeh O, et al. Large-scale wholegenome sequencing reveals the genetic architecture of primary membranoproliferative GN and C3 glomerulopathy. J Am Soc Nephrol. 2020;31:365-73.

32. Zhang T, Lu J, Liang S, Chen D, et al. Comprehensive analysis of complement genes in patients with atypical hemolytic uremic syndrome. Am J Nephrol. 2016:43:160-9.

33. Zhai $Y$, Meng $S$, Zhu L, et al. Rare variants in the complement factor h-related protein 5 gene contribute to genetic susceptibility to $\lg A$ nephropathy. J Am Soc Nephrol. 2016;27:2894-905.

34. Zipfel PF, Wiech T, Stea ED, et al. CFHR gene variations provide insights in the pathogenesis of the kidney diseases atypical hemolytic uremic syndrome and C3 glomerulopathy. J Am Soc Nephrol. 2020;31:241-56.

\section{Publisher's Note}

Springer Nature remains neutral with regard to jurisdictional claims in published maps and institutional affiliations.
Ready to submit your research? Choose BMC and benefit from:

- fast, convenient online submission

- thorough peer review by experienced researchers in your field

- rapid publication on acceptance

- support for research data, including large and complex data types

- gold Open Access which fosters wider collaboration and increased citations

- maximum visibility for your research: over $100 \mathrm{M}$ website views per year

At BMC, research is always in progress.

Learn more biomedcentral.com/submissions 